\title{
BMJ Open Quality Implementation of depression and anxiety screening in patients undergoing radiotherapy
}

\author{
Adam L Holtzman, ${ }^{1}$ Deidre B Pereira, ${ }^{2}$ Anamaria R Yeung ${ }^{1}$
}

To cite: Holtzman AL, Pereira DB, Yeung AR. Implementation of depression and anxiety screening in patients undergoing radiotherapy.BMJ Open Quality 2018;7:e000034. doi:10.1136/ bmjoq-2017-000034

Received 22 February 2017 Revised 13 February 2018 Accepted 24 March 2018

\section{Check for updates}

'Department of Radiation Oncology, University of Florida College of Medicine, Gainesville, Florida, USA

${ }^{2}$ Department of Clinical and Health Psychology, University of Florida College of Public Health and Health Professions, Gainesville, Florida, USA

Correspondence to Dr Anamaria R Yeung; areyna@ufl.edu

\begin{abstract}
Anxiety and depression are disabling, underdiagnosed issues that affect the management of oncology patients. Until recently, there has been no standard protocol for screening and treating these ailments at our cancer centre. The purpose of this institutional review board-approved study was to analyse the implementation of a screening tool in our clinical workflow with the aim of screening over $90 \%$ of patients and increasing referrals to mental health services by $50 \%$.
\end{abstract}

\section{PROBLEM}

Oncologic patients undergoing radiation therapy are at high risk for mental health disorders such as anxiety and depression. Evidence shows that early treatment and diagnosis of anxiety and depression reduces clinical morbidity and improves patient outcomes. $^{2}$ Historically, our cancer centre did not have a routine process for screening or referring patients to clinical health psychology, potentially putting our patients at risk for underdiagnosis and undertreatment of these ailments.

To address these issues, our cancer centre started an initiative to screen patients for depression and anxiety. To determine the programme's efficacy, the department of radiation oncology piloted a version of this intervention before implementation throughout the entire cancer centre.

\section{BACKGROUND}

Depression and anxiety are among the most prevalent and disabling medical conditions in the USA, yet they are often underdiagnosed in the general population, much less among those with cancer. A recent publication by the US Preventive Services Task Force reflects this issue and attempts to address it by recommending routine screening for depression in the general adult population, provided there are adequate systems in place to ensure accurate diagnosis, effective treatment and appropriate follow-up. ${ }^{2}$ Such screening has led to decreased clinical morbidity and improved clinical outcomes. ${ }^{2}$ Additionally, there is no evidence that screening for depression or anxiety causes harm. ${ }^{3}$

Patients with cancer are at an increased risk of depression and anxiety compared with the general population. Not only do they face difficult treatment courses and sometimes end-of-life issues, but they may also be subjected to the stressors of living away from home, oncological pain, inability to care for themselves or costly medical procedures. ${ }^{4-10}$ In fact, in a survey of over 100 patients with non-small cell lung cancer, almost $50 \%$ self-reported their depression. ${ }^{10}$ We propose that by initiating screening and appropriate treatment, we will better diagnose and manage these illnesses.

\section{BASELINE MEASUREMENT}

Under institutional review board approval, baseline measurements were obtained by retrospectively reviewing all patients treated in the previous 5 months prior to the intervention. Information obtained included age, gender, diagnosis, the intent of treatment such as curative or palliative, history of mental health illness and current medications.

Of the identified 58 individuals, the median age was 65 (range 27-89), and $67 \%$ $(n=39)$ were treated with curative-intent radiotherapy (RT). The primary sites of disease included lung ( $72 \%$; $n=42)$, gynecologic $(22 \% ; n=13)$ and other $(6 \% ; n=3)$. No patient was screened for anxiety or depression at baseline, nor were any of these patients referred to mental health services by the radiation oncology department. Conversely, the medical oncology department referred three patients seen in radiation oncology to mental health services. Although not our primary study metric, since this study was not designed to assess the adequacy of medication use in patients with known mental health issues, we retrospectively reviewed, and reported baseline data show that 53\% $(n=31)$ of patients had been on an anxiolytic 
$(n=15)$ or antidepressant $(n=24)$ within the year prior to beginning RT.

\section{DESIGN}

At the start of RT, nurses administered the Patient Health Questionnaire-4 (PHQ-4). The PHQ-4 was chosen because it is a four-question validated screening tool to assess both anxiety and depression in the outpatient setting and because versions of this test have been used to assess patients with cancer, which represents our target population. ${ }^{11-13}$ In regard to diagnostic testing, our oncology social workers conducted a thorough diagnostic assessment in which they performed evaluations for depression and anxiety along with other aetiologies, such as an adjustment disorder or substance abuse and, when necessary, placed a referral to clinical psychology. Patients with a total score of 6 or greater were automatically referred to an oncology social worker. The social worker assessed for other aetiologies, such as an adjustment disorder or substance abuse and, when necessary, placed a referral to clinical psychology. Study aims were to screen over $90 \%$ of patients for depression or anxiety and to increase referrals to mental health services by $50 \%$.

Detailed medication history was also obtained to determine the anxiolytic or antidepressant medications at the start of treatment for all patients and subsequently added to the electronic medical record. We updated this list on a weekly basis and recorded when a new medication was started.

\section{STRATEGY}

Several Plan, Do, Study, Act (PDSA) cycles were used during the development phase of this quality improvement project:

\section{PDSA cycle 1}

We implemented the intervention with the plan to refer all patients with a positive screening directly to clinical psychology for assessment.

\section{PDSA cycle 2}

After the initial implementation, we found it difficult for the clinical psychology service to manage the increase in referrals. The decision was made to instead have the patients first referred to an oncology social worker for an initial evaluation. They were then assessed more thoroughly for depression and anxiety along with adjustment disorders or substance abuse, and, if deemed appropriate by the social worker, patients would be further referred to clinical psychology.

\section{PDSA cycle 3}

After discussion with treating physicians, we realised that the information about the screening was not reaching the physicians. We developed a clear pathway for this information to be seen and acted on by the treating radiation oncologist.

\begin{tabular}{|c|c|c|}
\hline Characteristics & $\begin{array}{l}\text { Preintervention } \\
(\mathrm{n}=58)\end{array}$ & $\begin{array}{l}\text { Postintervention } \\
(n=119)\end{array}$ \\
\hline $\begin{array}{l}\text { Median patient } \\
\text { age (range) }\end{array}$ & 65 (27-89) years & 65 (26-89) years \\
\hline \multicolumn{3}{|c|}{ Gender (men/women) } \\
\hline Male & 31 patients & 58 patients \\
\hline Female & 27 patients & 61 patients \\
\hline \multicolumn{3}{|l|}{ Primary site } \\
\hline Lung & $72 \%$ (42 patients) & $66 \%$ (79 patients) \\
\hline Gynecologic & 22\% (13 patients) & 26\% (31 patients) \\
\hline Other & $6 \%$ (3 patients) & $8 \%$ (9 patients) \\
\hline \multicolumn{3}{|c|}{ Intent of treatment } \\
\hline Curative & $67 \%$ & $68 \%$ \\
\hline Palliative & $33 \%$ & $32 \%$ \\
\hline
\end{tabular}

\section{PDSA cycle 4}

Interim analysis was performed 3 months after study initiation showing that we were able to effectively implement screening into the clinical workflow without noticeable reductions in workflow or patient care. Following an additional 4 months of study with the continued positive results, we have since implemented anxiety and depression screening prior to the start of radiation treatments for every patient in our clinic, and this process has been integrated into our electronic medical record system.

\section{PDSA cycle 5}

To maintain sustainability of the programme, we have recently hired a dedicated social worker for the department of radiation oncology to facilitate diagnostic screenings and evaluations. We have also partnered with the medical oncology department and cancer centre to standardise the screening tools used in these evaluations. Owing to discussions with our clinical health psychologists, we replaced the PHQ-4 with the PHQ-2 alongside the perceived stress scale (PSS) to ensure that the entire cancer centre is using an analogous system, thereby improving the sustainability of the programme.

\section{RESULTS}

Patient characteristics comparing baseline measurements and following intervention are shown in table 1. Results of our study aims were conducted at two separate time intervals following the intervention.

\section{First postintervention measurement}

The first analysis was performed 3 months after implementing the study intervention. The median age of the initial 36 patients enrolled following implementation was 68 years (range $38-82$ years). Overall, 66\% $(n=23)$ were treated with curative-intent RT, and primary disease sites included lung $(53 \% ; n=19)$, gynecologic $(31 \% ; n=11)$ and 
Number of Patients Screened Positive

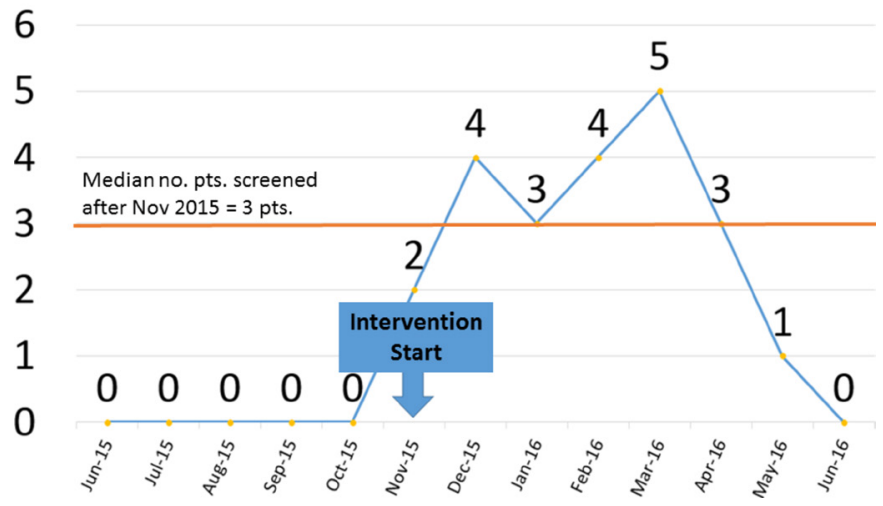

Figure 1 Run chart illustrating the number of positive screening tests over time.

other $(16 \% ; n=6)$. Of these individuals, $97 \%(35 / 36)$ were screened for anxiety and depression using the PHQ-4, of which $25 \%(\mathrm{n}=9)$ screened positive for the composite score. Further, $11 \%(\mathrm{n}=4)$ of the patients screened or $33 \%$ of those with a positive screening test were referred to clinical psychology. Interim analysis showed that we were able to effectively implement screening into the clinic without any noticeable reductions in workflow or patient care.

\section{Second postintervention measurement}

Following an additional 4 months of the intervention, we accrued 83 more patients for a total of 119 patients. Overall, 92\% (109/119) of patients were screened for anxiety and depression using the PHQ-4 following the intervention. Of those screened, 21\% (23/109) were positive for the composite score of either anxiety or depression. Figure 1 depicts a run chart illustrating the number of patients who screened positive over time. Five per cent $(n=6)$ of the patients screened or $22 \%$ of those with a positive screening test were referred to clinical psychology. Of note, 16 patients who screened positive on PHQ-4 testing and were offered referral to clinical health psychology declined further intervention for the following reasons: not interested at this time $(n=14)$, good social support $(\mathrm{n}=1)$ and had tried in the past but did not work as well as hoped $(n=1)$. The reason for one patient not being referred was not documented.

Interestingly, prior to screening, $45 \% \quad(n=54)$ of patients were already taking either only an anxiolytic $(n=31)$ or antidepressant $(n=41)$ before starting RT. Of the remaining 65 patients, $22 \%(n=14)$ were started on an anxiolytic $(n=7)$ or antidepressant $(n=12)$ during RT.

\section{LESSONS}

By implementing a simple screening tool for anxiety and depression in the radiation oncology clinic, we were able to screen $92 \%$ of patients, with $21 \%$ screening positive for the PHQ-4 composite score. This screening programme resulted in referring $26 \%$ of patients who screened positive for anxiety and/or depression to clinical psychology.
In the study period prior to the intervention, no patients were referred to clinical psychology from the radiation oncology department. Based on the encouraging results of this study, we have since implemented anxiety and depression screening prior to the start of radiation treatments for every patient in our clinic and integrated the process into our electronic medical records system. We have also hired an oncology social worker dedicated to the radiation oncology department rather than relying on a shared oncology social worker. A dedicated social worker for this programme is of particular importance now that more providers and staff will be using this system. We have also partnered with the medical oncology department and cancer centre to standardise the screening tools used. As mentioned, we replaced the PHQ-4 with the PHQ-2 alongside the PSS. The PSS replaces two questions regarding anxiety in the PHQ-4, and the PSS is also a common screening tool used with patients with cancer. ${ }^{14}$ Although our processes have changed since our initial study, the current system incorporates processes that the medical oncology department has used successfully in the past and ensures the viability and robustness of the programme across the entire cancer centre.

The most significant lesson from this intervention relates to the integration of care between physicians and support staff. This intervention was driven by the nurses and social workers who are conducting the assessments and educating patients on mental health disorders. The system still relies heavily on supporting social work and clinical psychology staff and would have proved difficult to implement without them. In fact, the US Preventive Services Task Force notes in their updated guidelines that screening must be performed with adequate systems in place to ensure that once patients screen positive, they are appropriately diagnosed and treated. Additionally, there remains to be a stigma associated with seeking counselling. The overwhelming majority of those patients who were recommended for further counselling declined stating that they were not interested at that time.

\section{LIMITATIONS}

Our intervention waslimited to one service including only thoracic and gynecological disease sites. The results of the intervention might vary with the introduction of patients with different types of malignancies. Long-term follow-up will also be required to assess whether patients can maintain successful continuation of needed psychological services. For instance, most of our patients are travelling from far distances and therefore might have difficulty receiving counselling by our institution's clinical psychology services. Therefore, either integration or transfer of services from our tertiary care centre to a local provider would be necessary. An alternative would be coordinating oncological follow-up with clinical psychology to minimise travel burden. Ultimately, this would remain an issue that requires further study. 


\section{CONCLUSION}

Following implementation of the PHQ-4 screening tool as part of the new patient workflow, $92 \%$ of patients were screened for anxiety and depression. Of those screened, $21 \%$ had a positive test for depression, anxiety or both. This resulted in $5 \%$ of all patients or $26 \%$ of the screen-positive patients being referred to clinical psychology, a significant improvement compared with $0 \%$ before the intervention. The screening and referral process was easily integrated into the new patient workflow and electronic medical record system, although the next step in implementation should focus on barriers to receiving psychological counselling as $70 \%$ of patients declined referral.

Acknowledgements Jodi Williams, LPN; Debra Hutchinson, RN; Karen Schroeder, RN; Lynn Searles, RN.

Contributors ALH and DBP acquired, analysed and interpretation of the data and drafted the manuscript. ARY designed the project and revised the manuscript. All authors approved the final version of manuscript.

Funding The authors have not declared a specific grant for this research from any funding agency in the public, commercial or not-for-profit sectors.

Competing interests None declared.

Patient consent Not required.

Ethics approval This project was approved by our institutional review board.

Provenance and peer review Not commissioned; externally peer reviewed.

Open Access This is an Open Access article distributed in accordance with the Creative Commons Attribution Non Commercial (CC BY-NC 4.0) license, which permits others to distribute, remix, adapt, build upon this work non-commercially, and license their derivative works on different terms, provided the original work is properly cited and the use is non-commercial. See: http://creativecommons.org/ licenses/by-nc/4.0/

(C) Published by the BMJ Publishing Group Limited. For permission to use (where not already granted under a licence) please go to http://www.bmj.com/company/ products-services/rights-and-licensing/

\section{REFERENCES}

1. Kroenke K, Spitzer RL, Williams JB, et al. An ultra-brief screening scale for anxiety and depression: the PHQ-4. Psychosomatics 2009;50:613-21.

2. Siu AL, Bibbins-Domingo K, Grossman DC, et al. Screening for Depression in Adults: US Preventive Services Task Force Recommendation Statement. JAMA 2016;315:380-7.

3. Rost K, Nutting P, Smith J, et al. Improving depression outcomes in community primary care practice: a randomized trial of the quEST intervention. Quality Enhancement by Strategic Teaming. J Gen Intern Med 2001;16:143-9.

4. Löwe B, Wahl I, Rose M, et al. A 4-item measure of depression and anxiety: validation and standardization of the Patient Health Questionnaire-4 (PHQ-4) in the general population. J Affect Disord 2010;122:86-95.

5. Osório FL, Lima MP, Chagas MH. Screening tools for psychiatry disorders in cancer setting: caution when using. Psychiatry Res 2015;229:739-42.

6. Mitchell AJ, Chan M, Bhatti $\mathrm{H}$, et al. Prevalence of depression, anxiety, and adjustment disorder in oncological, haematological, and palliative-care settings: a meta-analysis of 94 interview-based studies. Lancet Oncol 2011;12:160-74.

7. Park EM, Rosenstein DL. Depression in adolescents and young adults with cancer. Dialogues Clin Neurosci 2015;17:171-80.

8. Miller K, Massie MJ. Depression and anxiety. Cancer J 2006;12:388-97.

9. Butow P, Price MA, Shaw JM, et al. Clinical pathway for the screening, assessment and management of anxiety and depression in adult cancer patients: Australian guidelines. Psychooncology 2015;24:987-1001

10. Shi Y, Gu F, Hou LL, et al. Self-reported depression among patients with non-small cell lung cancer. Thorac Cancer 2015;6:334-7.

11. Temel JS, Greer JA, Muzikansky A, et al. Early palliative care for patients with metastatic non-small-cell lung cancer. N Engl J Med 2010;363:733-42.

12. Thekkumpurath P, Walker J, Butcher I, et al. Screening for major depression in cancer outpatients: the diagnostic accuracy of the 9-item patient health questionnaire. Cancer 2011;117:218-27.

13. Hegel MT, Collins ED, Kearing S, et al. Sensitivity and specificity of the distress thermometer for depression in newly diagnosed breast cancer patients. Psychooncology 2008;17:556-60.

14. Herschbach P, Book K, Brandl T, et al. Psychological distress in cancer patients assessed with an expert rating scale. Br J Cancer 2008;99:37-43. 